\begin{tabular}{|c|c|}
\hline $\begin{array}{l}\text { Chemistry of } \\
\text { Metals and Alloys }\end{array}$ & 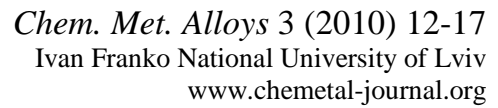 \\
\hline
\end{tabular}

\title{
Synthesis of borosilicate MFI type zeolite using different aging techniques
}

\author{
Maryam ABRISHAMKAR ${ }^{1}$, Seyed Naser AZIZI ${ }^{2} *$, Hossein KAZEMIAN ${ }^{3}$ \\ ${ }^{1}$ Department of Chemistry, Islamic Azad University, Khozestan Science and Research Branch, Ahvaz, Iran \\ ${ }^{2}$ Analytical Division, Faculty of Chemistry, University of Mazandaran, Babolsar, Iran, P.O. Box: 47416-95447 \\ ${ }^{3}$ Department of Chemical and Biochemical Engineering, Faculty of Engineering, \\ The University of Western Ontario, London, Ontario, Canada N6A 5B9 \\ * Corresponding author.Tel.: +981125242002; e-mail: azizi@umz.ac.ir
}

Received February 14, 2010; accepted June 29, 2010; available on-line November 5, 2010

The isomorphic substitution of boron into ZSM-5 zeolite under static hydrothermal conditions was investigated. The evaluation of the hydrothermal synthesis of BZSM-5 was done by treating the synthesis mixture by different aging processes, namely, ultrasonic, static, stirring, and microwave-assisted aging prior to the conventional hydrothermal treatment. The synthetic processes with different techniques of aging prior to the onset of conventional hydrothermal crystallization were compared with a process without any prior aging. The obtained results show that ultrasonic and microwave assisted aging shorten the crystallization time and alter the crystal size and the morphology of the obtained products. The characteristics of the synthesized products were determined by FT-IR, XRD and SEM techniques.

\section{BZSM-5 / Microwave / Ultrasonic / Aging / Crystallization time}

\section{Introduction}

Zeolites are a class of microporous crystalline aluminosilicate materials with pores of the same size as small molecules. The ZSM-5 zeolite is important as catalyst from an industrial point of view, due to its thermal stability, unique structure, shape selective property, acidity, and applications in oil industry for refinery, petro-chemical manufacturing and environmental catalysis. Isomorphous substitutions of metal for silicon $(\mathrm{Si})$ in the lattice structure of zeolites have been studied to modify the physico-chemical properties [1-4]. In borosilicate MFI type zeolites, aluminum is substituted by boron, which was studied by Taramasso et al. for the first time (see [5-7]).

Conventional heating is a routine method for zeolite synthesis, with heat transferred to the synthetic mixture by conduction, convection and radiation. In this case, the increase in temperature is relatively slow and less uniform due to the presence of a temperature gradient.

The use of a microwave-assisted hydrothermal method could provide an efficient way to rapidly synthesize various zeolite crystals and rationally control their particle size distribution, morphology and phase purity. In this technique, the microwave radiation is absorbed directly and uniformly; thus the increase in temperature is rapidly and uniformly distributed within the entire synthetic mixture, which significantly reduces the synthesis time from several days to several hours [8-10]. The dielectric heating of microwave irradiation not only facilitates a more uniform reaction without thermal gradients, but also leads to elevated reaction temperatures for size focusing due to its special selective heating [11]. Brar et al. [12] reported that the microwave radiation led to a narrow particle size distribution and a small crystal size. Additionally, the microwave synthesis is also expected to prepare some zeolite materials with special morphology.

The use of power ultrasound to enhance chemical reactivity is called sonochemistry. Applying ultrasound was shown to effect changes in the rates, yields and properties of the product in several processes $[13,14]$. Ultrasound can enhance and alter dissolution processes, chemical reactions, nucleation and growth of precipitates $[15,16]$. Ultrasound and microwave-assisted techniques are rapid for preparing more homogenous and stable material compared to conventional hydrothermal synthesis techniques.

On the base of our knowledge, the synthesis of BZSM-5 under conventional hydrothermal conditions is time consuming. In this paper, we report an efficient ultrasound-assisted and time dependent aging procedure for shortening the crystallization time of the BZSM-5 synthesis. The synthesis mixture was treated with several aging procedures prior to conventional heating, in order to study their effect on the 
crystallization period. It was observed that with all the techniques the synthesized product was BZSM-5 zeolite, however, the crystallization period was reduced in the microwave- and ultrasound-assisted synthesis of BZSM-5. Infra-red spectroscopy, X-ray diffraction (XRD) and particle size measurements were carried out to substantiate the results.

\section{Experimental \\ 2.1. Synthesis}

Tetraethylorthosilicate (TEOS), boric acid, sodium hydroxide, and tetrapropylammonium bromide (TPABr) as organic reagent, were the chemical reagents used, purchased from Merck Company. The B-ZSM-5 samples were prepared using a synthetic solution with initial composition of $2.1 \mathrm{Na}_{2} \mathrm{O}: \mathrm{B}_{2} \mathrm{O}_{3}$ : $2.4 \mathrm{SiO}_{2}$ : 4 TPABr: $1050 \mathrm{H}_{2} \mathrm{O}$. TEOS was hydrolyzed in the sodium hydroxide solution, then TPABr as a structure forming agent was added to the above solution under stirring. Boric acid was poured into the silicate solution and the obtained mixture was stirred to obtain a clear solution. The borosilicate solution was processed by the different aging methods, then transferred to a Teflon-lined stainless steel autoclave and the hydrothermal treatment was carried out for the appropriate time ( $18 \mathrm{~h}$ to 4 days) in a preheated convection oven at $180^{\circ} \mathrm{C}$ under static conditions. The obtained zeolite powder was rinsed with distilled water and calcined for $5 \mathrm{~h}$ at $550^{\circ} \mathrm{C}$.

\subsection{Apparatus}

The X-ray powder diffraction (XRD) patterns were collected on a GBC MMA instrument. The patterns were obtained with Ni-filtered $\mathrm{CuK \alpha}$ radiation $(\lambda=1.5418 \AA$ ) at $35.4 \mathrm{kV}$ and $28 \mathrm{~mA}$ with a scanning speed of $2 \theta=10^{\circ} \mathrm{min}^{-1}$. FT-IR spectra were recorded at room temperature using a FT-IR spectrometer (Vector 22-Bruker), over the range $400-2000 \mathrm{~cm}^{-1}$ with a resolution of $2 \mathrm{~cm}^{-1}$ on $\mathrm{KBr}$ pellets. Scanning electron microscopy was performed on selected samples to determine the crystallite size and morphology using a JEOL JXA-840 SEM instrument.

\section{Results and discussion \\ 3.1. The effect of different aging on the FT-IR spectra of as-synthesized BZSM-5}

The IR spectra of all as-synthesized B-ZSM-5 samples are typical of pentasil zeolites. The IR spectra of samples with an initial synthetic solution $\mathrm{SiO}_{2} / \mathrm{B}_{2} \mathrm{O}_{3}$ ratio of 2.4, prepared under different aging processes prior to conventional heating, are illustrated in Fig. 1. The distinct bands at 445, 790 and the saturated region $1000-1300 \mathrm{~cm}^{-1}$ are characteristic of $\mathrm{SiO}_{4}$ tetrahedra. The vibrational band at $546 \mathrm{~cm}^{-1}$ confirms the presence of five-member rings of pentasil structure in
MFI type zeolites. The as-synthesized samples exhibit two weak absorption peaks at 670 and $920 \mathrm{~cm}^{-1}$. In Bandyopadhyay and Camblor's works, the bands at 670 and $920 \mathrm{~cm}^{-1}$ are attributed to the Si-O-B symmetric bending and stretching vibrations of the tetra-coordinated framework boron, respectively [17]. These bands do not appear in Al-ZSM-5 and silicalite-1, and their intensity is attributed to the content of boron incorporated in the zeolite framework. For the sample without aging (Fig. 1(a)) this band is distinctive; for the other samples on which aging treatments were done, the intensity decreases (Fig. 1(b-d)).

\subsection{Effects of aging techniques on the crystallization time and morphology of the synthesized zeolite}

The synthesis of B-ZSM-5 from a solution with an initial composition with a ratio of $2.1 \mathrm{Na}_{2} \mathrm{O}: \mathrm{B}_{2} \mathrm{O}_{3}$ : $2.4 \mathrm{SiO}_{2}$ : 4 TPABr: $1050 \mathrm{H}_{2} \mathrm{O}$ by the conventional hydrothermal technique at $180^{\circ} \mathrm{C}$ for 5 days without use of any aging technique prior to hydrothermal treatment was checked by X-ray power diffraction (XRD). The XRD pattern of the obtained zeolite showed that after about 5 days at $180^{\circ} \mathrm{C}$ a highly crystalline B-ZSM-5 could be obtained.

Because of the long crystallization time of borosilicate MFI type zeolite, the synthesis of B-ZSM-5 was performed with the above composition of the initial mixture, and the effects of static, stirring, microwave and ultrasonic aging prior to conventional hydrothermal treatment on the crystallinity of the obtained B-ZSM-5 were investigated. The corresponding results under different aging conditions of the synthesis mixture are shown in Table 1. The illustration of such pre-treatment methods is shown in Fig. 2. From the obtained results, it can be concluded that the aging step plays an important role in shortening the crystallization time in synthesizing B-ZSM-5 zeolite. If no aging or stirring aging of the solution in the synthetic process was performed, crystallization times between 110 and $120 \mathrm{~h}$ would be required to obtain highly crystalline zeolite. In the cases of ultrasound-assisted aging for $0.5 \mathrm{~h}$ and microwave-assisted aging for $0.5 \mathrm{~h}$ and $1 \mathrm{~h}$ prior to heat treatment, the crystallization time could be efficiently shortened, to $24 \mathrm{~h}$ and $18 \mathrm{~h}$ by $0.5 \mathrm{~h}$ ultrasonic and by $1 \mathrm{~h}$ microwave-assisted aging, respectively. The $40 \mathrm{~h}$ static aging at room temperature followed by $72 \mathrm{~h}$ conventional treatment resulted in a zeolite with good crystallinity. These results show that the induction period and crystallization period were reduced after ultrasonic and microwave-assisted aging, because these pretreatments affect the depolymerization-polymerization equilibrium by increasing the concentration of the soluble crystalline species needed for the formation the BZSM-5 zeolite.

A comparative view of different aging techniques on the crystalline properties of the obtained products 


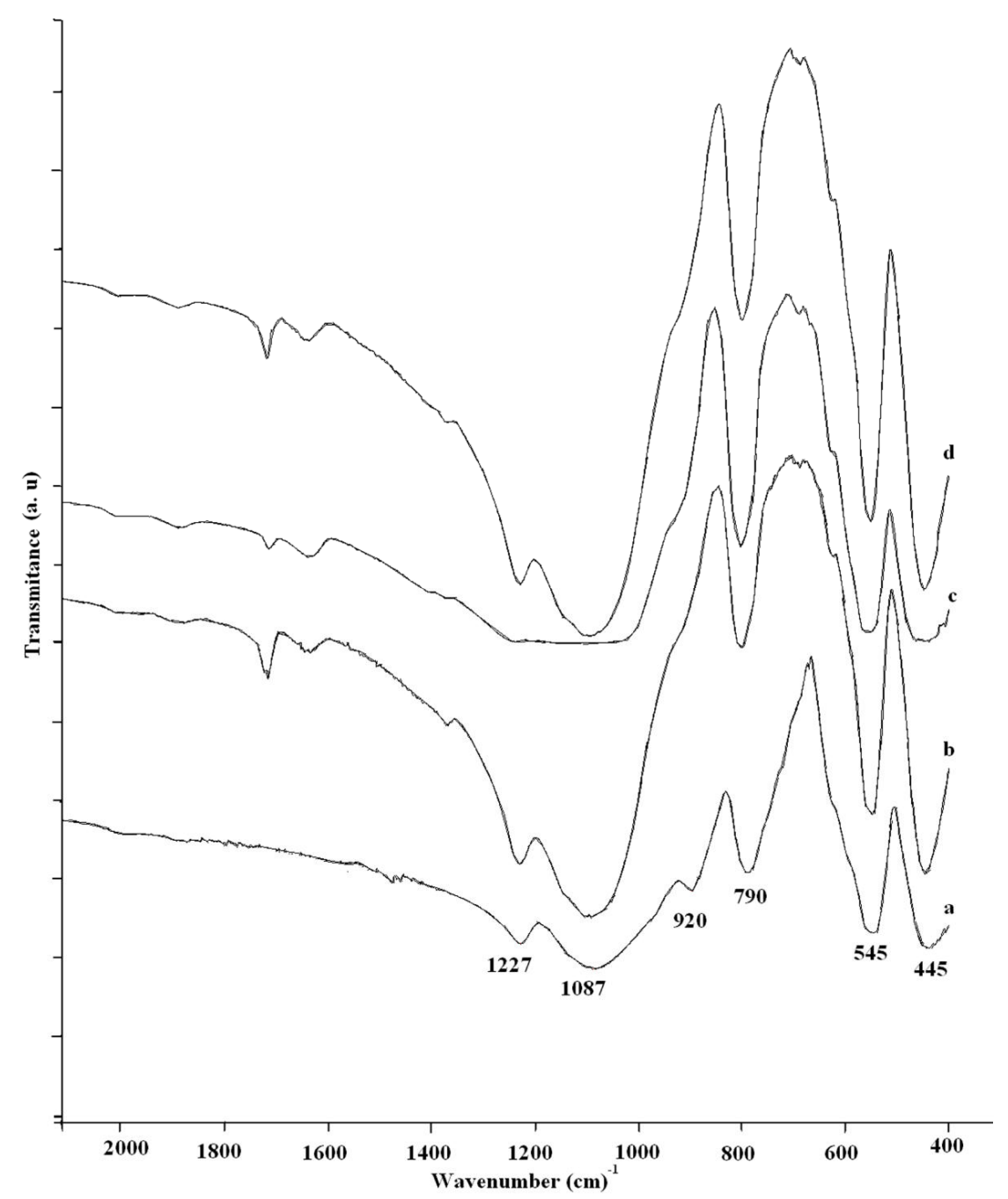

Fig. 1 FT-IR spectra of synthesized BZSM-5 samples with initial synthetic solution $\mathrm{SiO}_{2} / \mathrm{B}_{2} \mathrm{O}_{3}$ ratio of 2.4 prepared under different aging treatments: (a) without aging, (b) microwave-assisted aging for $0.5 \mathrm{~h}$, (c) ultrasonic aging for $0.5 \mathrm{~h}$, and (d) static aging for $40 \mathrm{~h}$, prior to conventional treatment at $180^{\circ} \mathrm{C}$.

Table 1 Synthesis conditions and average crystal size.

\begin{tabular}{c|c|c|c}
\hline Aging method & Aging time (h) & $\begin{array}{c}\text { Time of hydrothermal } \\
\text { treatment }(\mathbf{h})\end{array}$ & $\begin{array}{c}\text { Average volume of } \\
\text { sample crystals }\left(\boldsymbol{\mu ~ m}^{\mathbf{3}}\right)\end{array}$ \\
\hline No aging & - & 120 & 60 \\
\hline Stirring aging & 0.5 & 110 & 60 \\
\hline \multirow{3}{*}{ Microwave-assisted } & 0.5 & 18 & 8 \\
& 0.5 & 24 & 8 \\
& 0.5 & 48 & 8 \\
& 0.5 & 72 & 8 \\
& 0.5 & 96 & 6 \\
\hline \multirow{3}{*}{ Ultrasound-assisted } & 1 & 18 & 50 \\
& 0.5 & 18 & 50 \\
& 0.5 & 24 & 50 \\
& 0.5 & 48 & 50 \\
& 0.5 & 72 & 55 \\
\hline \multirow{2}{*}{ Static aging } & 0.5 & 96 & 60 \\
& 40 & 72 & 60 \\
\hline
\end{tabular}




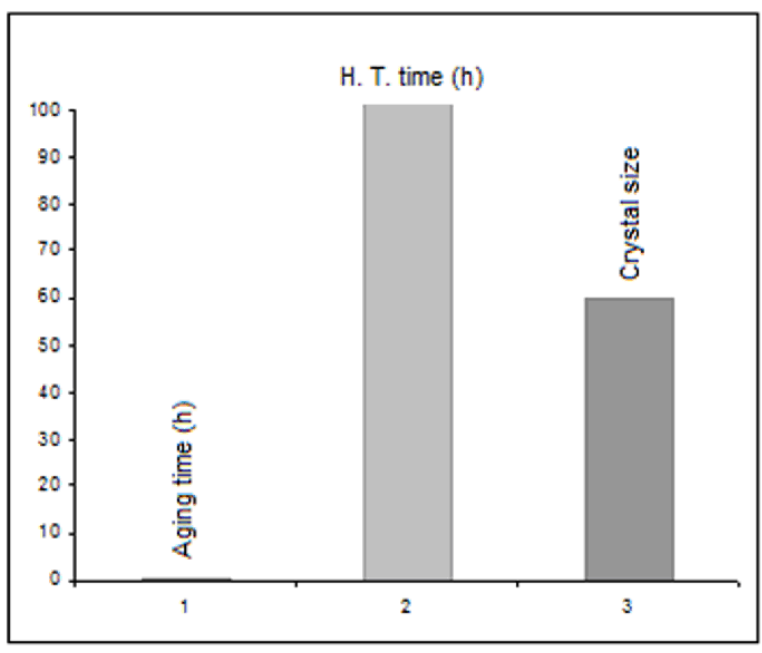

(a) Stirring aging technique

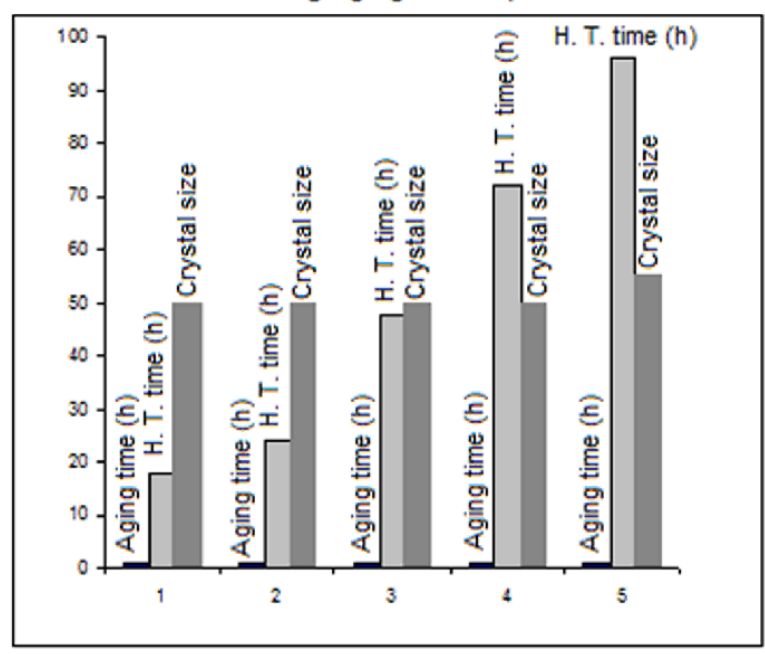

(c) Ultrasond-assisted aging technique

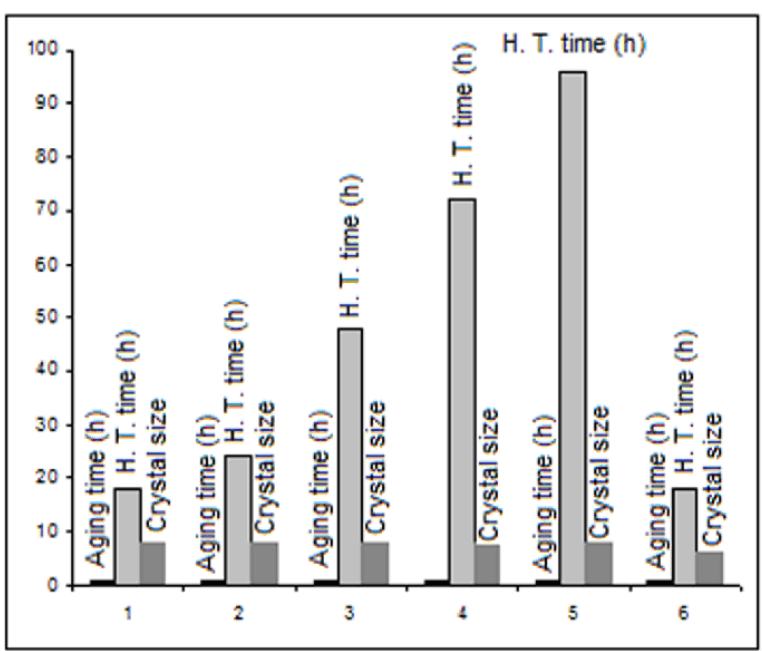

(b) Microwave-assisted aging technique

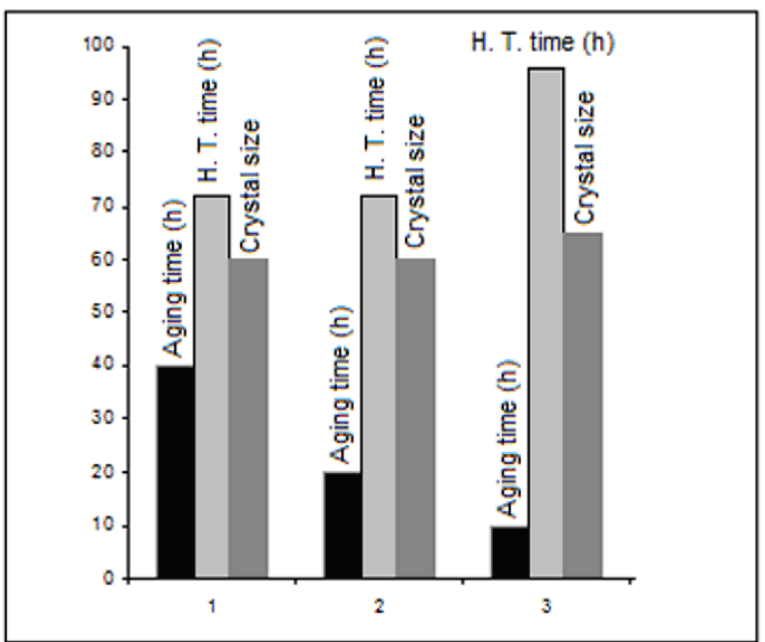

(d) Static aging technique

Fig. 2 Various aging methods, related conventional hydrothermal times and obtained crystal sizes.

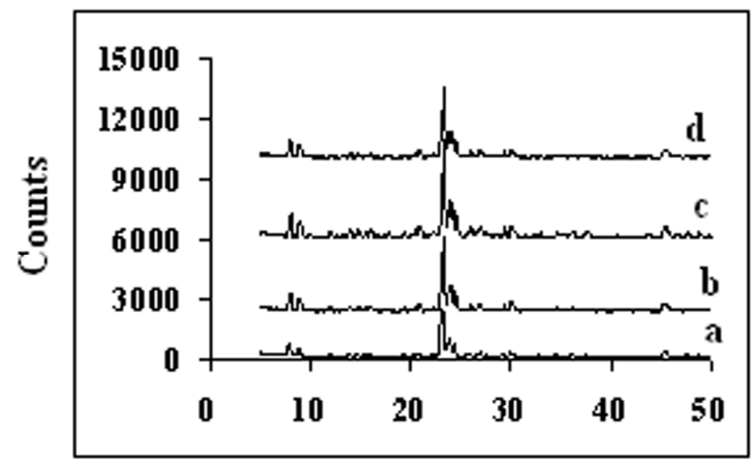

2 theta (deg)

Fig. 3 X-ray diffraction patterns showing the effect of different aging on the formation of BZSM-5: (a) stirring, (b) microwave-assisted, (c) ultrasonic aging for $0.5 \mathrm{~h}$, and (d) static aging for $40 \mathrm{~h}$, prior to conventional treatment at $180^{\circ} \mathrm{C}$. is shown in Fig. 3. As mentioned before, microwave and ultrasonic aged samples (Fig. 3(b,c)) showed good crystallinity in lesser crystallization period with respect to samples that were prepared after pretreatment by stirring or static aging (Fig. 3(a,d)). The SEM images of as-synthesized BZSM-5 samples under different aging techniques are illustrated in Fig 4.It can be seen that the shapes of the particles are very similar to each other after different kinds of aging, but the crystal size of the samples is different. The crystals of BZSM-5 resembled cubic structures in all aging procedures. In the case of microwaveassisted aging, the SEM images revealed a significant influence on the crystal size of the product because such aging could decrease the particle size to around $10 \mu \mathrm{m}^{3}$.

\section{Conclusion}

Pure and highly crystalline BZSM-5 zeolite was successfully synthesized by ultrasonic, microwaveassisted and static aging procedures. It was found that 

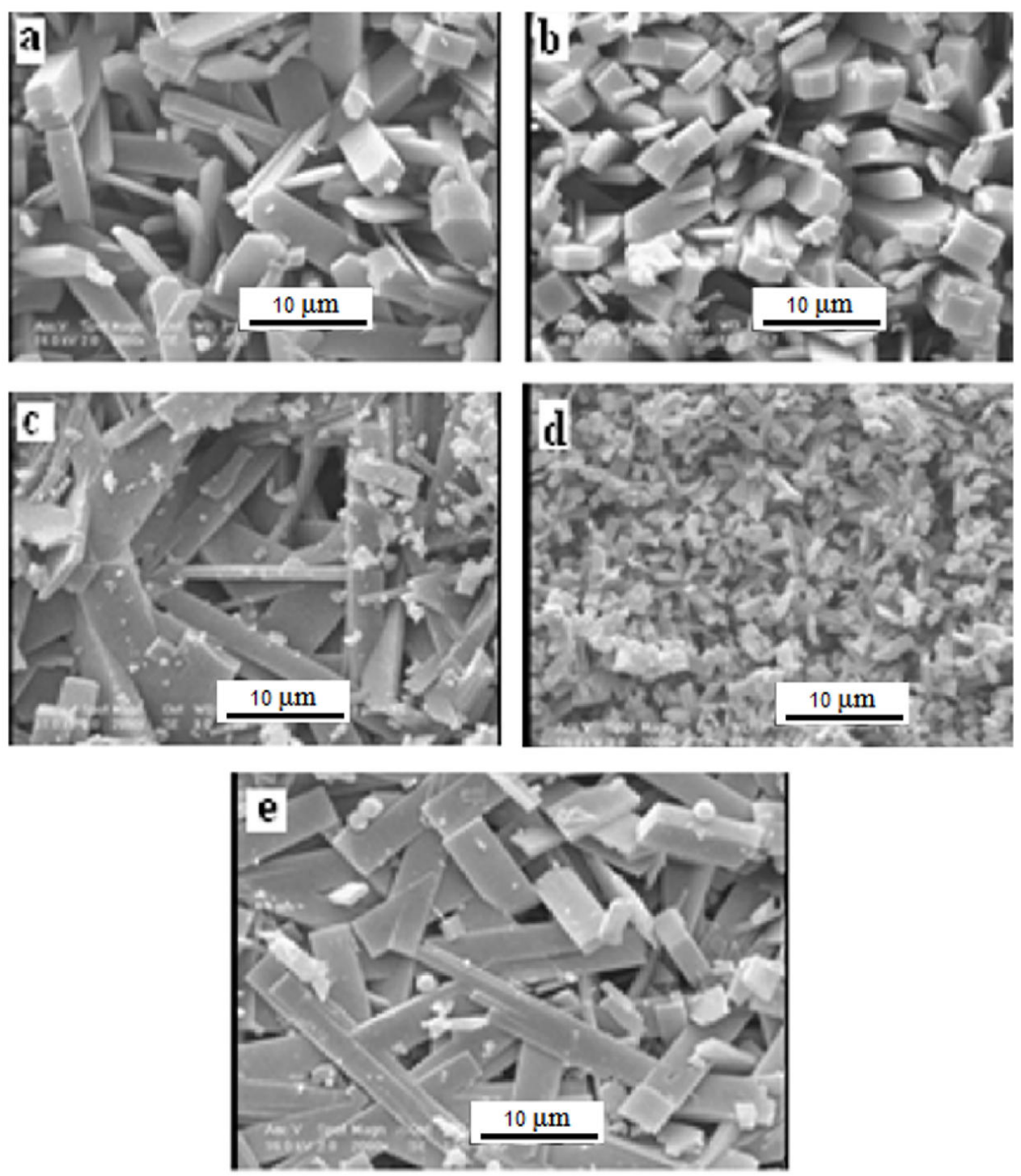

Fig. 4 Effect of different aging on the crystal morphology and size of products (a) without aging, (b) with stirring aging for $0.5 \mathrm{~h}$, (c) with static aging for $40 \mathrm{~h}$, (d) with microwave-assisted aging for $0.5 \mathrm{~h}$, and (e) with ultrasonic-assisted aging for $0.5 \mathrm{~h}$, followed by hydrothermal synthesis at $180^{\circ} \mathrm{C}$.

the aging of the synthetic mixture resulted in reducing the induction period and the duration of crystallization. By varying the aging techniques we have been able to experimentally improve the nucleation of borosilicate MFI type zeolite, which requires a long crystallization period under the conventional method without any pre-treatment. As one can see, aging enhances the rate of nucleation and shortens the nucleation period during the crystallization process. It was found that extending the static aging period from $10 \mathrm{~h}$ to $40 \mathrm{~h}$ decreases the crystallization time from $96 \mathrm{~h}$ to $72 \mathrm{~h}$, and increases the crystallinity of the obtained product. We have also demonstrated that the morphology of BZSM-5 obtained after different aging processes does not change and only after using the microwave-assisted aging procedure the crystal size was reduced.

\section{Acknowledgement}

The authors would like to thank Ellen Vuosalo Tavakoli (University of Mazandaran) for final editing of the English text.

\section{References}

[1] C. Naccache, Y.B. Tarrit, In: F.R. Ribeiro, A.E. Rodrigues, L.D. Rollmann, C. Naccache (Eds.), 
Zeolite Science and Technology, Martinus Nijhoff Publishers, The Hague, 1984.

[2] W. Song, R.E. Justice, C.A. Jones, V.H. Grassian, S.C. Larsen, Langmuir 20 (2004) 8301.

[3] S.P. Yuan, J.G. Wang, Y.W. Li, H. Jiao, J. Phys. Chem. A 106 (2002) 8167.

[4] H. Kalipcilar, S.K. Gade, R.D. Noble, J.L. Falconer, J. Membr. Sci. 210 (2002) 113.

[5] V. Sundaramurthy, N. Lingappan, J. Mol. Catal. A: Chem. 160 (2000) 367.

[6] G.P. Heitmann, G. Dahlhoff, J.P.M. Niederer, W.F. Holderich, J. Catal. 194 (2000) 122.

[7] W. Zhou, S.Y. Zhang, X.Y. Hao, H. Guo, C. Zhang, Y.Q. Zhang, S.I. Liu, J. Solid State Chem. 179 (2006) 855.

[8] Y. Hu, C. Liu, Y. Zhang, N. Ren, Y. Tang, Microporous Mesoporous Mater. 19 (2009) 306.

[9] W.C. Conner, G. Tompsett, K.H. Lee, K.S. Yngvesson, J. Phys. Chem. B 108 (2004) 13913.
[10] O.G. Somani, A.L. Choudhari, B.S. Rao, S.P. Mirajkar, Mater. Chem. Phys. 82 (2003) 538.

[11] J.A. Gerbec, D. Magana, A. Washington, G.F. Strouse, J. Am. Chem. Soc. 127 (2005) 15791.

[12] T. Brar, P. France, P.G. Smirniotis, Ind. Eng. Chem. Res. 40 (2001) 1133.

[13] S.H. Jhung, K.C. Yoo, Y.K. Hwang, J.S. Chang, Bull. Korean Chem. Soc. 28 (2007) 2401.

[14] O. Andac, S.M. Telli, M. Tatlier, A. ErdemSenatalar, Microporous Mesoporous Mater. 88 (2006) 72.

[15] Y.S. Li, Q.G. Yin, Ultrasonic Sonochemistry, Science Press, Beijing, 1995.

[16] B. Wang, J. Wu, Z.Y. Yuan, N. Li, S. Xiang, Ultrason. Sonochem. 15 (2008) 334.

[17] M.A. Camblor, A. Corma, J.P. Pariente, J. Chem. Soc., Chem. Commun. 7 (1992) 360. 